\title{
1 Shared and unique properties of place cells in anterior cingulate cortex
} and hippocampus

${ }^{\dagger}$ Corresponding author:

18 Yasunori Hayashi, MD, PhD

E-mail: yhayashi-tky@umin.ac.jp

Keywords: Anterior cingulate cortex, hippocampus, place cells, memory consolidation, $\mathrm{Ca}^{2+}$ imaging

Conflict of interest statement:

YH was partly supported by Fujitsu Laboratories and Dwango.

Present address:

28 Alessandro Luchetti

29 University of California, Los Angeles, CA 90095, USA

30 Hajime Hirase

31 University of Copenhagen, Center for Translational Neuromedicine, 2200 Copenhagen N., 


\section{Abstract}

In the brain, spatial information is represented by neurons that fire when an animal is at specific locations, including place cells in hippocampus and grid cells in entorhinal cortex. But how this information is processed in downstream brain regions still remains elusive. Using chronic $\mathrm{Ca}^{2+}$ imaging, we examined the activity of neurons in anterior cingulate cortex (ACC), a brain region implicated in memory consolidation, and found neurons that fire in a manner consistent with the properties of place cells. While the ACC place cells showed stability, location and context specificity similar to the hippocampal counterparts, they also have unique properties. Unlike hippocampal place cells that immediately formed upon exposure to a novel environment, ACC place cells increased over days. Also, ACC place cells tend to have additional place fields whereas typical hippocampal place cells have only one. Hippocampal activity is required for the formation of ACC place cells, but once they are established, hippocampal inactivation did not have any impact on ACC place cell firing. We thus identified features of ACC place cells that carry spatial information in a unique fashion.

\section{Introduction}

Spatial navigation is an essential element of animal behavior that allows animals to forage, return home and avoid dangers. The hippocampus plays a crucial role in this process. It bears neurons called place cells that fire when an animal is located in a particular position of an environment but not in others, providing an allocentric cognitive map of a space [1-4]. Multimodal inputs including visual, tactile, olfaction, auditory, proprioception, and other sensory information are integrated to form the place cells in hippocampus [1-4]. It was also shown that the spatial representation of hippocampal place cell is initially dynamic but gradually stabilized [5-8]. The stabilization selectively occurs at a location with motivational (reward) and environmental (landmark) salience and results in an over-representation in these locations, indicating that the place cells' activity is not merely a representation of the space but also its cognitive value. In this way, hippocampal place cells are well characterized from years of studies [1-4]. However, how spatial information is further processed and chronically represented in downstream brain regions has not been fully elucidated at cellular resolution [5].

We decided to focus on anterior cingulate cortex (ACC), a part of medial prefrontal cortex (mPFC) implicated in memory consolidation [9-11]. Suppression of ACC impaired the recall of the remote but not of the recent memory[11]. This is in contrast to hippocampus, which is required for the recall of only recent but not remote memory [11, 12]. ACC receives a direct input from hippocampus [13] and also from striatum, amygdala and retrosplenial cortex, thereby serving as an 
integration center of wide variety of sensory and motivational events [14, 15]. Recent work has revealed neurons with spatially specific firing in mPFC including ACC. This spatial firing is regulated by the environmental and task context, consistent with the spatial coding [16-19]. Lesion of the hippocampus abolishes the place code activity in the mPFC indicating that mPFC is situated in downstream of hippocampus for spatial coding [20]. However, how the spatial encoding of ACC neurons develops has not been fully examined.

We, therefore, chronically monitored the activity of neurons in ACC while animals navigate through a space. To this end, we used a head-mount miniaturized fluorescence microscope [5] to image $\mathrm{Ca}^{2+}$-responses of excitatory neurons in ACC over days from freely moving mice. We found neurons that fire in a manner consistent with the properties of place cells (ACC place cell). ACC place cell showed similar properties with hippocampal place cell such as increase in stability, reliability and context specificity after repeated exposure to the same environment over days. Bayesian decoding verified that those cells indeed carry positional information. On the other hand, we found several properties unique to ACC place cells. The fraction of ACC place cells increased as the task was repeated. ACC neurons tend to have multiple place fields, which might represent association of more than one location in a context. Finally, the formation of the ACC place cells requires hippocampal activity but once established, the cells become independent of hippocampus. Thus, while ACC place cells share properties with hippocampal place cells, they have unique features and mechanism of generation.

\section{RESULTS}

\section{ACC has place cells similarly to hippocampus}

For $\mathrm{Ca}^{2+}$ imaging, we used TRE-G-CaMP7-T2A-DsRed $2 \times$ CaMKII $\alpha-$ tTA double transgenic mice, that coexpress G-CaMP7 and DsRed2 in excitatory neurons [8]. To image ACC, a gradient reflective index (GRIN) lens with a microprism attached at its end was implanted between the two cortical hemispheres so that the center of the tip of the microprism is at $0.5 \mathrm{~mm}$ anterior from bregma and $1.4 \mathrm{~mm}$ from the cortical surface and the prism faces the left ACC [21] (Fig. 1a and Supplementary Fig. 1a). The dorsal hippocampus was imaged by implanting a GRIN lens above the right dorsal hippocampus after removing a part of overlaying cortex as previously reported [5] (Fig. $1 \mathrm{~b}$ and Supplementary Fig. 1b). The activity of ACC layer 2/3 neurons or hippocampal CA1 pyramidal neurons was observed by using a head-mounted fluorescent microscope across multiple days from awake and behaving animals [5] (Fig. 1c).

The animals were placed first in a square track (one edge $50 \mathrm{~cm}$, context S1) with a wall installed at one of the corners, then in an open arena $(50 \times 50 \mathrm{~cm}$, context $\mathrm{O})$ with different wall 
patterns and scent, and finally in the original square track (context S1') for 10 min each with 5 min intermissions (Fig. 1d). In context S1, the animals ran back-and-forth between the two ends for food rewards, given when the animal reached one end. The animals moved faster and finished more laps over days (Fig. 1e), indicating that the animal became familiar with the context[11]. In the context O, the rewards were randomly thrown into the open arena to motivate animals to navigate around [22].

In both hippocampus and $\mathrm{ACC}$, we found neurons that fire in a manner consistent with the properties known for place cells, such as location specificity, reproducibility, and direction selectivity across laps (Fig. 1f and g). The proportion of hippocampal place cells did not change over days, consistent with previous results [5]. The proportion of ACC place cells in early phase (days 1-3) was smaller than in hippocampus (Fig. 1h) but increased as the task was repeated and reached the level comparable to hippocampus in the late phase (days 7-9). We also calculated the reliability index of each cell, an index of how reproducibly a cell fires in a specific location across multiple laps in a single session (Supplementary Fig. 2). In early sessions (days 1-3), the index was lower in ACC than in hippocampus, but gradually increased in the late sessions (days 7-9) though it never reached the level of hippocampus (Fig. 1i). This indicates that the firing reproducibility of ACC place cells is not as high as that of hippocampus place cells. We noticed that nearly a half of ACC neurons had extra place fields (Fig. 1j) whereas hippocampal neurons typically had only one place field. The number of extra place fields remained constant in both ACC and hippocampus during repeated training, indicating that this is an intrinsic difference between these two brain regions.

In order to test if these cells indeed encode spatial information, we used Bayesian decoding (Fig. 1k) by selecting the same number of the cells with the highest activity level from both ACC or hippocampus. The decoder often registered one end of the square track as the other end. This may reflect the fact that the ends are adjacent to each other, separated just by a wall. Nevertheless, the analysis confirmed that the ACC neurons carry positional information comparable to those in hippocampus (hippocampus vs ACC; $p=0.46$ (early), $p=0.201$ (middle), $p=0.79$ (late), One way ANOVA. Fig.1k). Therefore, we called these cells ACC place cells. 
a

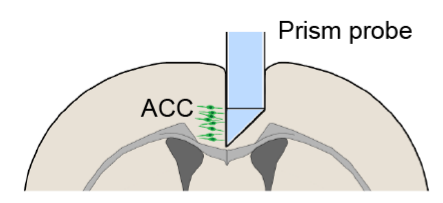

b

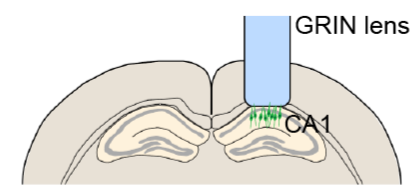

C

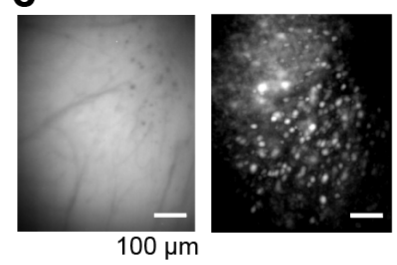

e Context $\mathrm{S1}$

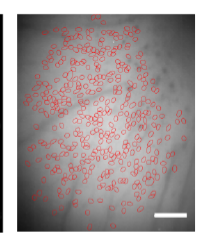

d
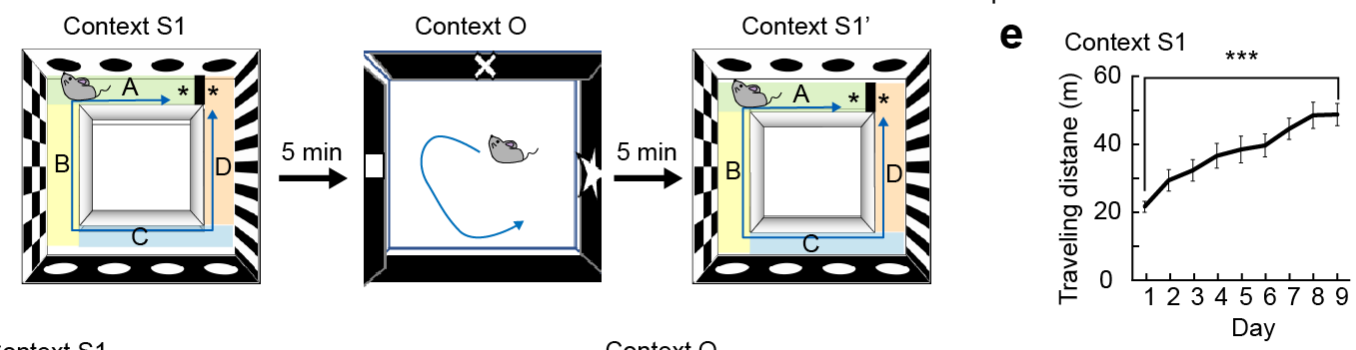

f
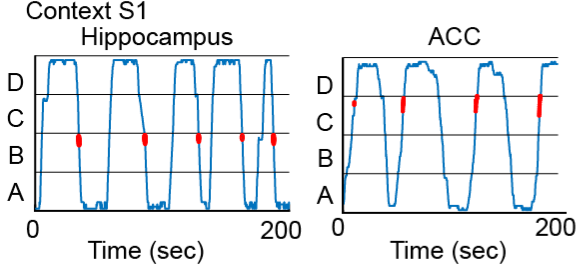

g Context $\mathrm{O}$
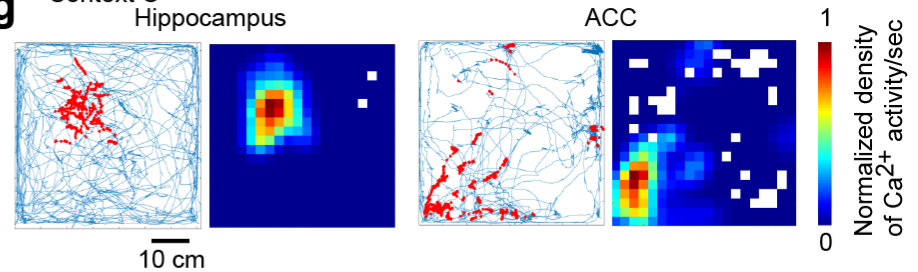

h

Hippocampus place cell

\section{i}
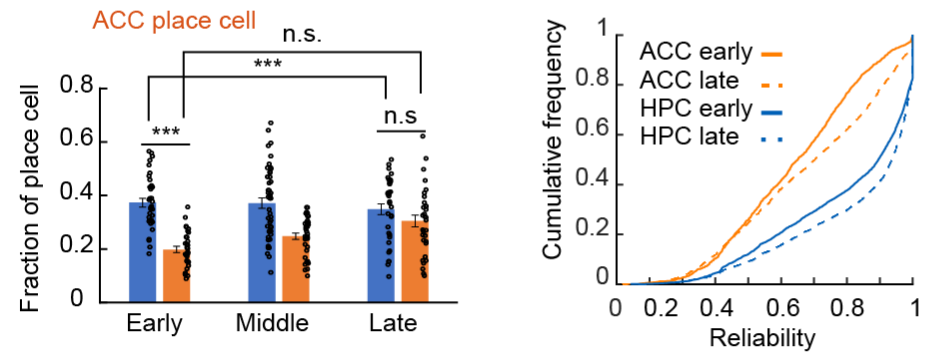

j
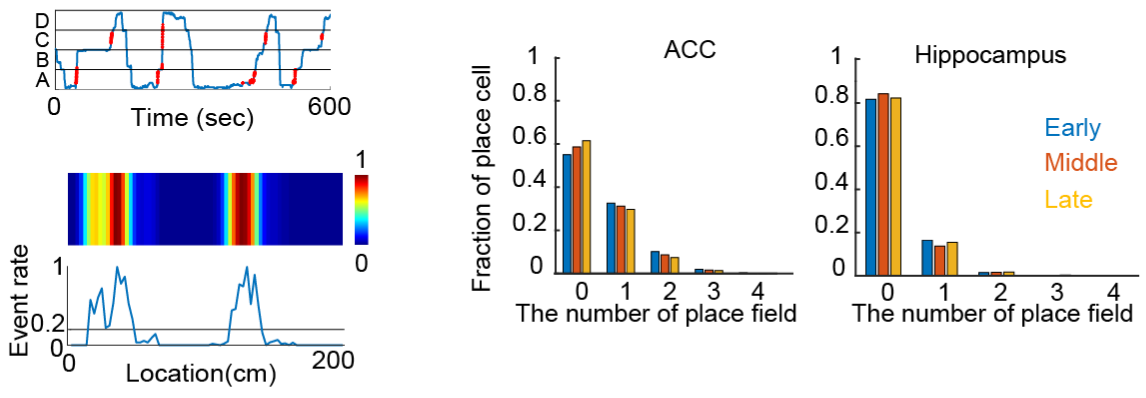

k
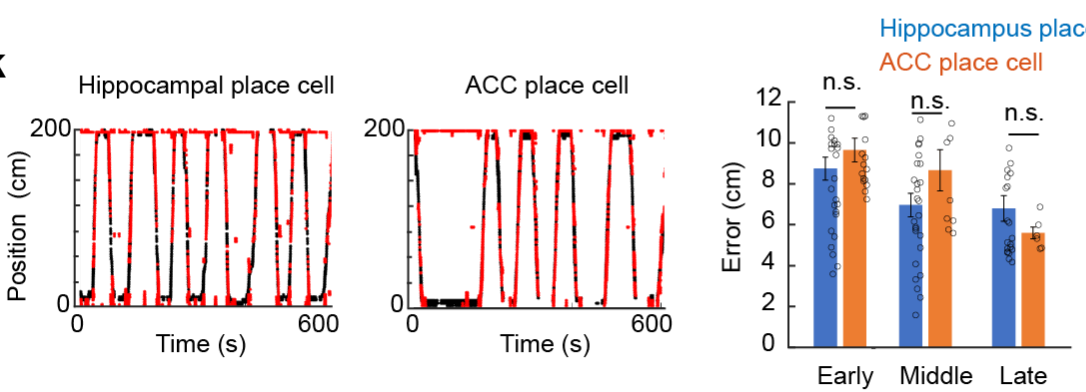

Bota et al. Fig. 1

Figure 1. The basic properties of ACC and hippocampal place cells. 
130 (a) Imaging of the ACC using a right angle microprism inserted into the fissure.

131 (b) Imaging of hippocampus by using a GRIN lens implanted above hippocampal CA1 layer.

132 (c) Example images of ACC neurons. Left, max intensity image. Blood vessels appear as shadows.

133 Middle, relative fluorescent change $(\Delta \mathrm{F} / \mathrm{F})$. Right, active cells (red circles) overlaid with the max

134 intensity image.

135 (d) Behavior paradigms. The square linear track (context S1 or S1') and the open arena (context O). 136 In one session, mice visited two distinct environments ( $\mathrm{S} 1 \rightarrow \mathrm{O} \rightarrow \mathrm{S}$ 1') each for $10 \mathrm{~min}$. Location of 137 reward in $\mathrm{S} 1$ was indicated by asterisks. In context $\mathrm{O}$, the reward was randomly thrown into the 138 arena. One set of experiment was conducted per day for 9 days while monitoring neuronal activity 139 in hippocampus or ACC.

140 (e) Behavioral changes induced by repeated training with context S1. Total traveled distance is 141 shown $\left(\mathrm{n}=9\right.$ mice). Day 1 vs day $9, \mathrm{p}=1.88 \times 10^{-8}$, one-way AVOVA. Graphs show means \pm 142 SEM.

143 (f) Example of place cells in hippocampus in ACC in context S1. Blue lines show the trajectory of 144 the mouse and red dots mark calcium events.

145 (g) Example of place cells in hippocampus and in ACC in context O. Left, blue lines show the mouse's trajectory and red dots mark calcium events. Right, Gaussian-smoothed density maps of calcium events, normalized by the mouse's occupancy time per unit area and the cell's maximum response.

(h) The fraction of ACC and hippocampal place cells relative to the number of total identified cells in context S1. Hippocampus early vs late $\mathrm{p}=0.69$, ACC early vs late $\mathrm{p}=2.9 \times 10^{-4}$, hippocampus early vs ACC early $\mathrm{p}=5.5 \times 10^{-10}$, hippocampus late vs ACC late $\mathrm{p}=0.079$; one-way ANOVA. N = 36 data (18 sessions x 2 running directions) in early, 46 data (23 sessions x 2 running directions) in middle, 34 data (17 sessions x 2 running directions) in late from 5 mice for hippocampus. $\mathrm{N}=32$ data (16 sessions x 2 directions) in early, 38 data (19 sessions x 2 directions) in middle, 32 data (16 sessions $\mathrm{x} 2$ directions) in late from 4 mice for ACC.

(i) Reliability of firing of ACC and hippocampus ('HPC') place cells in the early (days 1-3) and the late sessions (days 7-9). Reliability represents how reproducible is the during multiple laps in the same session (Fig. S2). Data were pooled from 3265 cells in the early and 3527 cells in the late sessions from 5 mice for hippocampus and 1206 cells in the early and 2494 cells in the late sessions from 4 mice for ACC. Hippocampus early vs late $\mathrm{p}=1.61 \times 10^{-12}$; ACC early vs late $\mathrm{p}=7.23 \times 10^{-8}$ and ACC early vs hippocampus early $\mathrm{p}=1.403 \times 10^{-114}$ and ACC late vs hippocampus late $\mathrm{p}=3.68$ x $10^{-171}$, one-way ANOVA.

(j) Example of an ACC place cell with extra place fields in context S1. Left Top, mouse trajectory (blue) and calcium events (red). Left middle, linearized heat map of the event rate normalized by maximum activity. Left bottom, Normalized event rate in each spatial bin. Vertical line shows the threshold for criteria of place field. This cell has 2 place fields. Right, number of place field of hippocampus and ACC place cells in the early (days 1-3), middle (days 4-6) and late (days 7-9) 
sessions. $\mathrm{N}=5161$ place cells in early, 5018 place cells in middle, 3842 place cells from 5 hippocampus group mice. $\mathrm{N}=2453$ place cells in early, 2812 place cells in middle, 2972 place cells from 4 ACC group mice.

(k) Bayesian decoding of the mouse trajectory (red dots) and actual position (black curves) from hippocampus and ACC place cells. Average median errors of the early (days 1-3), middle (days 4-6) and late (days 7-9) sessions. Not significant by Wilcoxon rank sum test. $\mathrm{P}=0.46$ (early), $\mathrm{p}=0.201$ (middle), $\mathrm{p}=0.79$ (late). $\mathrm{N}=28$ (hippocampus) and 16 (ACC) sessions in early sessions; 27 (hippocampus) and 9 (ACC) sessions in the middle sessions, 21 (hippocampus) and 6 (ACC) sessions in the late sessions.

\section{Stability of hippocampus and ACC place cells between sessions}

We then compared the activity of each cell between two sessions in identical or distinct contexts to see whether they switched their encoding mode between sessions (Fig. 2a). When the S1 and $\mathrm{S} 1$ ' sessions interleaved by a session $\mathrm{O}$ were compared, $66.1 \pm 5.5 \%$ of ACC place cells in context $\mathrm{S} 1$ still behaved as place cells in the context S1' (Fig. 2b). In contrast, only $7.0 \pm 1.4 \%$ of ACC place cell in context $\mathrm{S} 1$ behaved as a place cell in context $\mathrm{O}$. The rest of the $\mathrm{S} 1$ place cells were either unclassified $(84.7 \pm 2.1 \%)$ or not active $(8.3 \pm 2.4 \%)$. This proportion of encoding mode was comparable to the proportion of all cell types in context $\mathrm{O}$ (place cells, $6.1 \pm 1.1 \%$; unclassified, 84.8 $\pm 2.2 \%$; not active $9.0 \pm 2.4 \% . \mathrm{p}=0.65,0.96,0.85, \mathrm{~N}=4$ mice; one-way ANOVA), indicating random reassignment of the place cells when mice moved to the other context. Likewise, place cells in context $\mathrm{O}$ converted their encoding in context $\mathrm{S} 1$ ', in a proportion not different from their proportion among all cells (Supplementary Fig. 3. Place cells, $30.6 \pm 7.0 \%$; unclassified cells $25.5 \pm$ $5.4 \%$, not active $44.0 \pm 8.1 \%$ versus all cells in context $\mathrm{S} 1^{\prime}$ : place cells, $33.2 \pm 4.6 \%$, unclassified cells, $29.0 \pm 3.6 \%$; not active, $37.8 \pm 3.0 \% ; \mathrm{p}=0.92,0.29,0.50 ; \mathrm{N}=4$ mice; one-way ANOVA). The same tendency was observed in hippocampal place cells. Therefore, this analysis indicates that the place cells in one context are randomly assigned either as place or non-place cells in different contexts and suggests that there is no particular subclass of neurons which preferentially become place cells in different contexts in both brain regions.

Finally, in order to quantitatively assess the change in the activity level of each cell, we defined the selectivity index by calculating the absolute of the difference between firing rate in two contexts normalized by the sum of firing rate in both contexts (Fig. 2c). This analysis revealed that both hippocampal and ACC place cells showed a significant increase in the selectivity over days, 
indicating that there was a significant differentiation in neuronal activity to one of the contexts during repeated exposure to two different contexts (Fig. 2c).

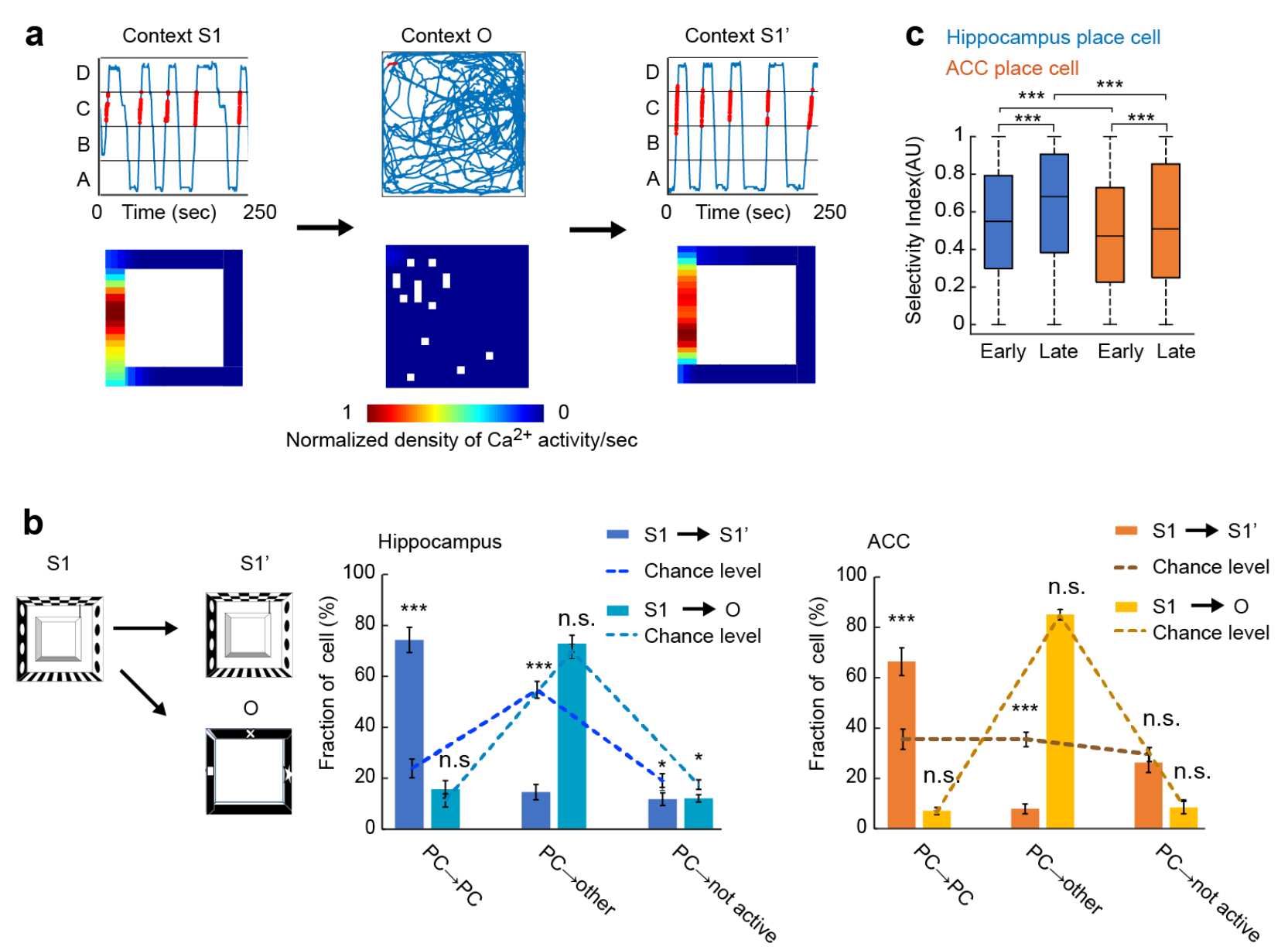

Bota et al. Fig. 2

Figure 2. Context specificity of ACC and hippocampal place cells.

206 (a) Representative activity of an ACC place cell during one session (context $\mathrm{S} 1 \rightarrow$ context

207 O $\rightarrow$ context S1'). Top, heat map for event rate of the cell. Bottom, mouse's trajectory (blue) and calcium events (red). Firing intensity is normalized by the maximum activity of the cell throughout the session.

210 (b) Comparison of place cell activity between two identical or different contexts. Encoding mode of ACC place cells in context S1 was examined in context S1' or context $\mathrm{O}$. ACC place cells in context S1 were classified in 3 groups "place cells (PC)", "other" and "not active" according to its activity pattern in context $\mathrm{S} 1$ ' or context $\mathrm{O}$. Figure shows the average fraction of cells in each group. Dotted lines show proportion of each class of cells in S1 or O, that serve as chance level. Compared with the chance level, hippocampus S1 to S1': place cells to place cells $\mathrm{p}=1.8 \times 10^{-8}$, place cells to other cell type $\mathrm{p}=1.2 \times 10^{-9}$, place cells to not active cells $\mathrm{p}=0.031$. Hippocampus $\mathrm{S} 1$ vs $\mathrm{O}$ : in the same order, $\mathrm{p}=0.42,0.58,0.018$. ACC S1 to S1': $\mathrm{p}=0.00027,1.3 \times 10^{-7}, 0.52$. ACC $\mathrm{S} 1$ to $\mathrm{O}: \mathrm{p}=0.65,0.96,0.85$. One-way ANOVA. $\mathrm{N}=4$ mice for ACC, $\mathrm{n}=5$ mice for hippocampus. 
219 (c) Selectivity index between context S1 versus context O. The index was pooled from 2045 220 hippocampal place cells (from 5 mice) and 931 ACC place cells (from 4 mice) in early phase (day 1221 3), 1655 hippocampal place cells (from 5 mice) and 1121 ACC place cells (from 4 mice) in late phase 222 (day 7-9). Whiskers show maximum and minimum value in each data set.

Long-term stability of place cells

We next examined whether these cells stably retain the same positional information on the track across sessions on the same day or different days. We first compared the place field in context $\mathrm{S} 1$ and $\mathrm{S} 1$ ' interleaved by a session in context $\mathrm{O}$ on the same day by calculating the population vector correlation. In hippocampus the correlation increased during the 9-day sessions (days 1-3 vs 7-9, p = $2.5 \times 10^{-3}$, one-way ANOVA). This is consistent with the previous results which showed hippocampal 231 place cells become stable after repeating the same task [8, 23]. Also in ACC, we saw an increase of 232 correlation between S1-S1' sessions over days (days 1-3 vs 7-9, p = $1.6 \times 10^{-7}$, one-way ANOVA).

233 We obtained essentially consistent result by calculating the ratio of cells with stable place fields (less 234 than $12.5 \mathrm{~cm}$ shift between S1-S1' sessions) among place cells in both hippocampus (44.05 $\pm 6.1 \%$ 235 of cells exhibited stable place field in days 1-3 and $64.7 \pm 5.5 \%$ in days 7-9, p $=0.0015$, one-way 236 ANOVA. Supplementary Fig.4) and ACC (28.7 $\pm 3.8 \%$ of cells exhibited stable place field in days $237 \quad 1-3$ and $58.1 \pm 3.6 \%$ in days 7-9, $\mathrm{p}=9.6 \times 10^{-6}$, one-way ANOVA).

238 We then attempted to obtain a more holistic view of stability over days by comparing S1 239 sessions across days (Fig. 3c-e). We also found that both the hippocampal and ACC place cells 240 showed a gradual stabilization during the sessions repeated over 9 days. These results showed that 241 the ACC place cells have similar properties to the hippocampal place cells with respect to the stability 242 (Hippocampus early vs late $\mathrm{p}=0.022$; ACC early vs late $\mathrm{p}=0.014$; ACC early vs hippocampus early $243 \mathrm{p}=0.93$; ACC late vs hippocampus late $\mathrm{p}=0.69$, one-way ANOVA). 
a
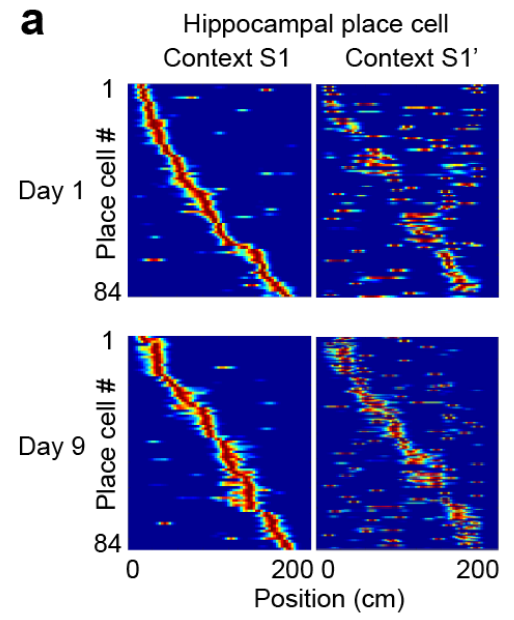

C
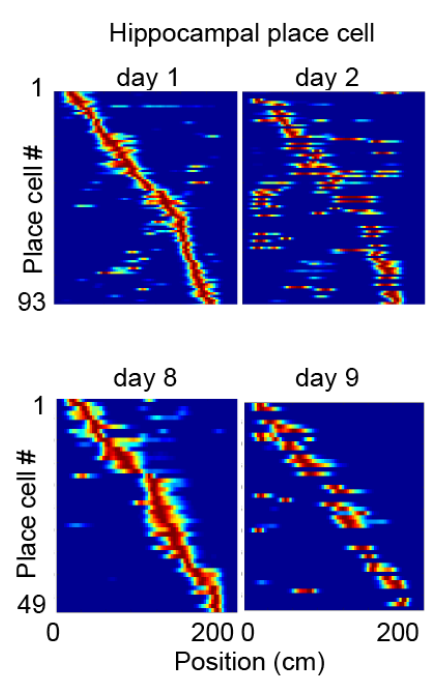
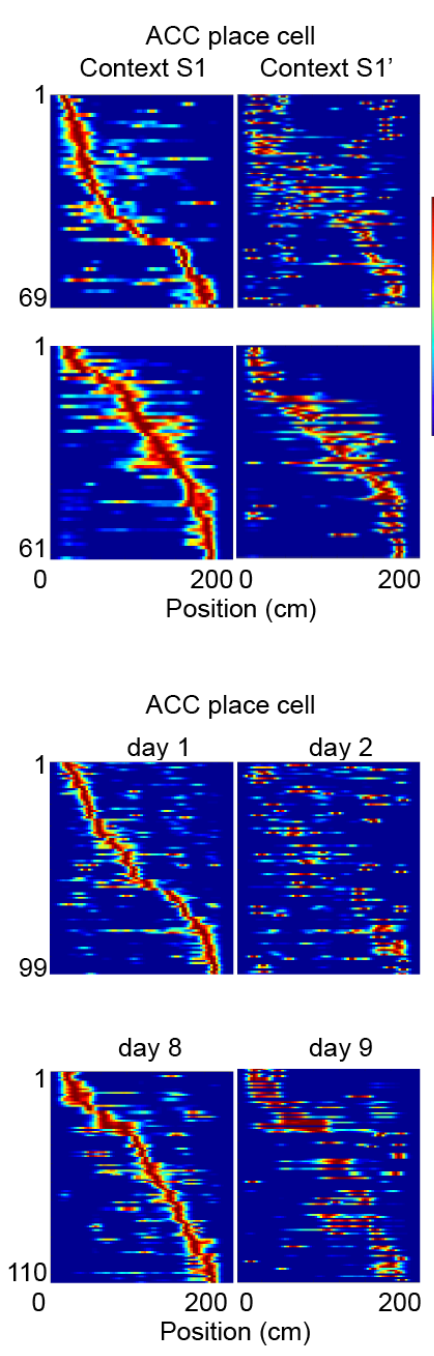

b
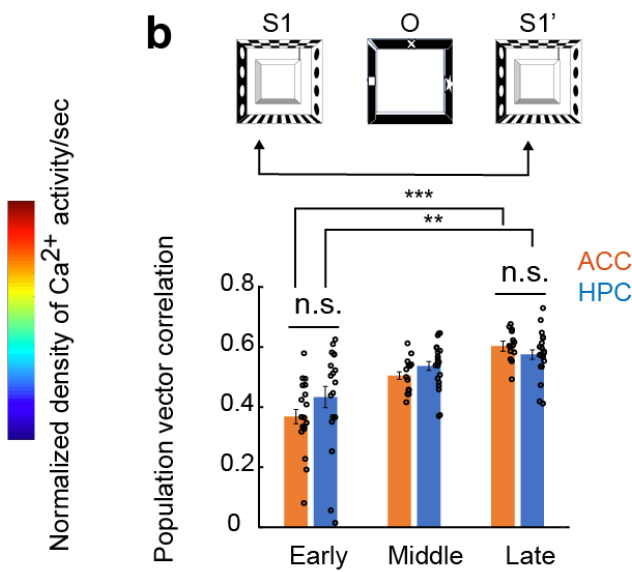

e

Day $\mathrm{n}$
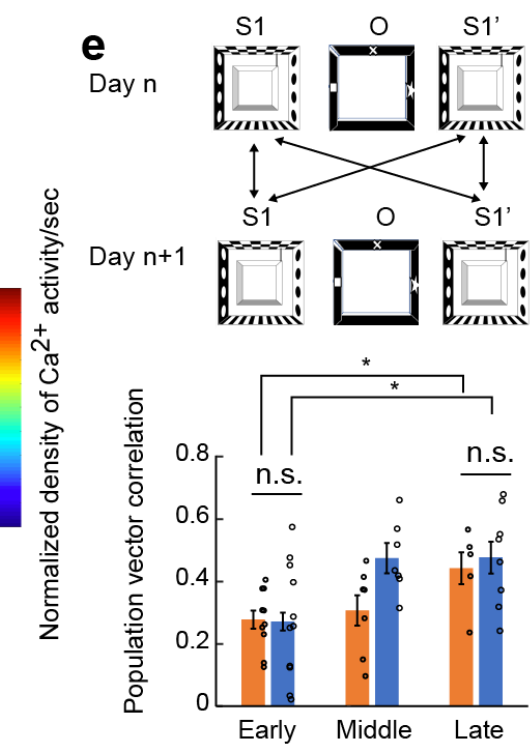

d

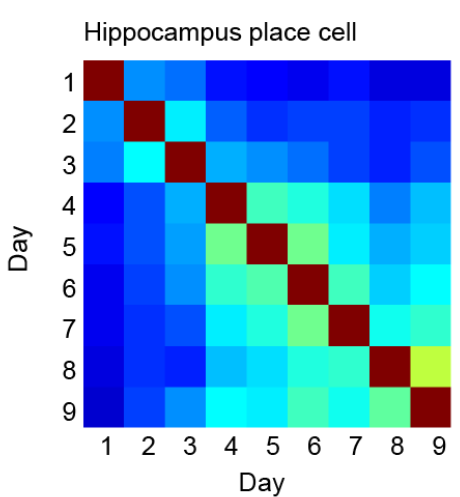

Day

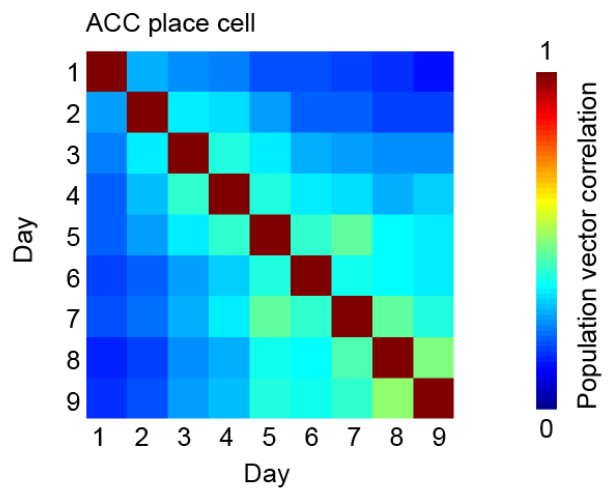

Bota et al. Fig. 3

246 Figure 3. Stability of ACC and hippocampal place cells across sessions.

(a) A comparison of place cell map between context $\mathrm{S} 1$ and $\mathrm{S} 1$ ' within the same session on day 1 and day 9. Place-field maps was ordered according to the place field centroid position in context S1. The maps were normalized by the maximum activity of each cell. 
(b) Population vector correlation of place cell representation in early, middle, and late sessions. Hippocampus early vs late $\mathrm{p}=2.5 \times 10^{-3}$; ACC early vs late $\mathrm{p}=1.6 \times 10^{-7}$; ACC early vs hippocampus early $\mathrm{p}=0.21$; ACC late vs hippocampus late $\mathrm{p}=0.24$, one-way ANOVA. $\mathrm{N}=18$ session pairs in early, 20 session pairs in middle, 20 session pairs in late for hippocampus. $\mathrm{N}=18$ session pairs in early, 14 session pairs in middle, 14 session pairs in late for ACC.

(c) A comparison of place cell map between context S1 and S1' in adjacent sessions.

(d) Heat map for population vector correlation of place cells in hippocampus and ACC between all pairs of sessions.

(e) Population vector correlation across early (day 1 vs day 2 to day 3 vs day4), and middle (day 4 vs day5 and day 5 vs day 6) and late (day 6 vs day 7 to day 8 vs day 9) sessions for both hippocampus and ACC. Hippocampus early vs late $\mathrm{p}=0.022$; ACC early vs late $\mathrm{p}=0.014$; ACC early vs hippocampus early $\mathrm{p}=0.93$; ACC late vs hippocampus late $\mathrm{p}=0.69$, one-way ANOVA. $\mathrm{N}=11$ session pairs in early, 7 session pairs in middle, 8 session pairs in late for hippocampus. $\mathrm{N}=10$ session pairs in early, 7 session pairs in middle, 5 session pairs in late for ACC.

ACC place cell activity requires hippocampus for formation but becomes independent after training

Finally, we examined whether the formation of ACC place cells requires hippocampal activity.

To this end, we inhibited the hippocampal neuronal activity by administering a Designer Receptor Exclusively Activated by Designer Drugs (DREADD) agonist 21 (D21) to a mouse expressing its cognate inhibitory receptor, hM4Di-mCherry, in dorsal hippocampus (Fig. 4a) [24]. The mice received D21 20 min before each behavior experiments for 9 days. Mice in saline hM4D(Gi) group received saline for 9 days and then received D21 on day 10 (Fig. 4b).

In the naïve, $\mathrm{D} 21$ naive or saline $\mathrm{hM} 4 \mathrm{D}(\mathrm{Gi})$ groups, there was an increase in fraction of ACC place cells during 9-day period (Fig. 4c). However, in the D21 hM4D(Gi) group, the fraction remained low compared with control animals (on day 9. Naive vs. D21 naive, $\mathrm{p}=0.83$; Naive vs. D21 hM4D(Gi), p = $1.9 \times 10^{-4}$; Naïve vs saline hM4D(Gi), p = 0.38. One way ANOVA. Fig. 4c). The stability of the map as assessed by the population vector correlation analysis between days 8 and 9 was also low stability in D21 hM4D(Gi) group compared saline hM4D(Gi) group (Fig. 4d. D21 hM4D(Gi) day 8 and 9 vs Saline hM4D(Gi) day 8 and 9, p = 0.023; D21 hM4D(Gi) day 8 and 9 vs Saline hM4D(Gi) day 9 and $10, \mathrm{p}=4.7 \times 10^{-6}$. One-way ANOVA). This indicates that the hippocampal activity is required for establishing and maintaining ACC place cell maps.

In contrast, when D21 was administered on day 10 in the saline $\mathrm{hM} 4 \mathrm{D}(\mathrm{Gi})$ group after the place cell map was already established, it did not have effect on the proportion of ACC place cells 
bioRxiv preprint doi: https://doi.org/10.1101/2021.03.29.437441; this version posted March 30, 2021. The copyright holder for this preprint (which was not certified by peer review) is the author/funder, who has granted bioRxiv a license to display the preprint in perpetuity. It is made available under aCC-BY-NC-ND 4.0 International license.

284 (Fig. 4c. $\mathrm{p}=0.96$, one-way ANOVA). Also, the population vector correlation between days 9 and 10 285 in the presence of D21 was not significantly different with days 8 and 9 when the same animals 286 received saline (Fig. 4d. $p=0.53$, one-way ANOVA). This indicates that ACC place cell map, once 287 established, no longer requires hippocampal neuronal activity for maintenance.

b

a

AAV8-CaMKIla-hM4D(Gi)-mCherry

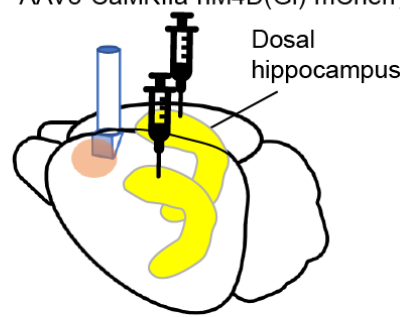

Naive

$$
\text { Day } 1
$$

$$
\text { Day } 9
$$

D 21

naive

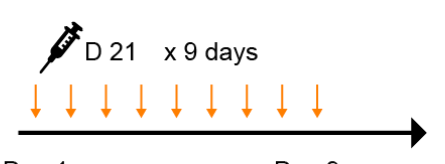

Day 1

Day 9

D 21

hM4D(Gi) D $21 \times 9$ days

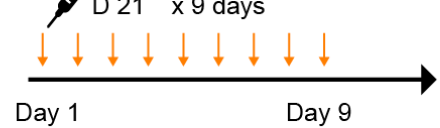

Saline

hM4D(Gi) 店 Saline $\times 9$ days

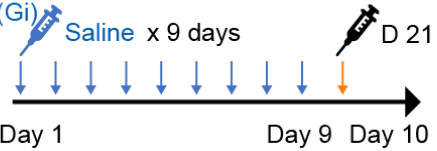

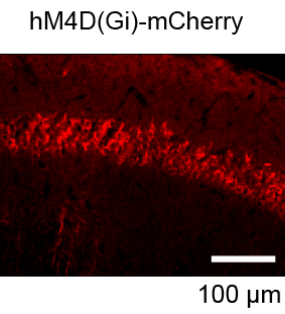

C

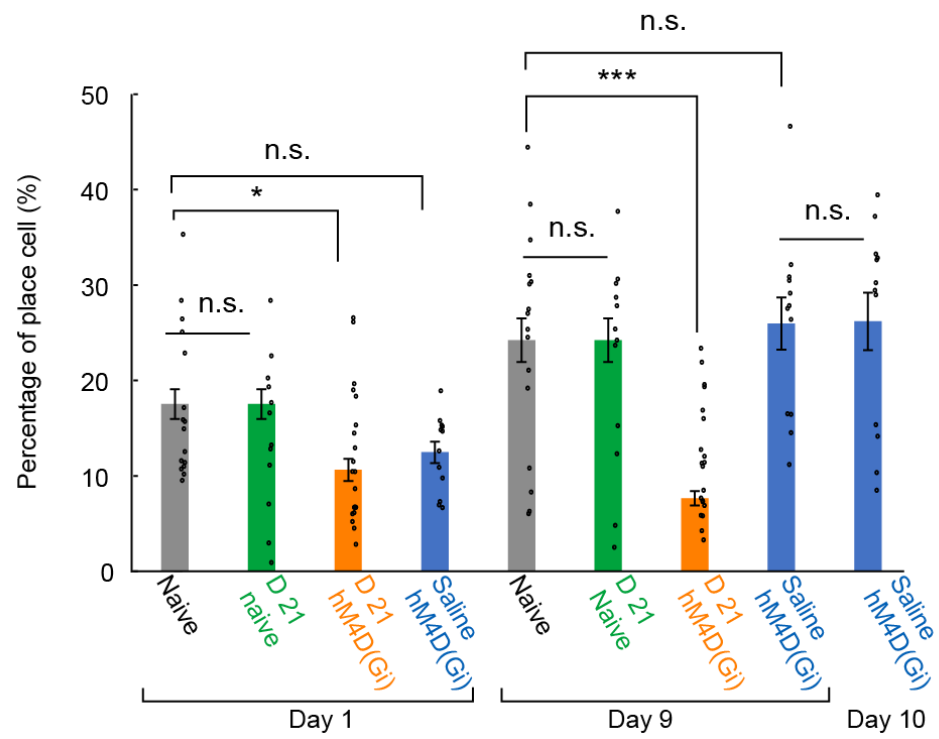

d

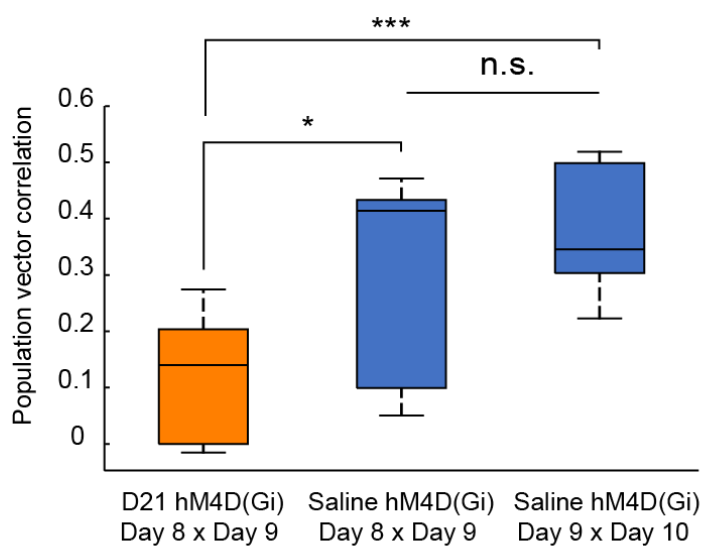

Bota et al. Fig.4 
Figure 4. The effect of chemogenetic inhibition of hippocampal excitatory neurons on ACC place cell.

(a) Representative mCherry image in TRE-GaMP7 x CaMKII $\alpha$-tTA double transgenic mice infected with AAV8-CaMKII $\alpha$-hM4D(Gi)-mCherry virus in hippocampi bilaterally. Scale bar $=$ $294100 \mu \mathrm{m}$.

(b) Experimental schedule.

(c) Percentage of ACC place cell in each context S1 and context S1'. In the bar graphs, circles represent individual trials from $\mathrm{S} 1$ or $\mathrm{S} 1$ '. (Naive $\mathrm{n}=16$ data ( 8 sessions $\mathrm{x} 2$ direction) from 4 mice, D21 naive $\mathrm{n}=12$ data ( 6 sessions $\mathrm{x} 2$ direction) from 3 mice, D21 hM4D (Gi) $\mathrm{n}=20$ data (10 sessions $x 2$ direction) from 5 mice, Saline $\mathrm{hM} 4 \mathrm{D}(\mathrm{Gi}) \mathrm{n}=12$ data (6 sessions $\mathrm{x} 2$ direction) from 3 mice). Day 1; naïve vs D21 naïve $\mathrm{p}=0.23$, naïve vs $\mathrm{D} 21 \mathrm{hM} 4 \mathrm{D}$ (Gi) $\mathrm{p}=0.033$, naïve vs saline hM4D Gi) $\mathrm{p}=0.054$. Day 9; naïve vs D21 naïve $\mathrm{p}=0.83$, naïve vs D21 hM4D (Gi) $\mathrm{p}=1.9$ x $10^{-4}$, naïve vs saline hM4D (Gi) $\mathrm{p}=0.38$, saline hM4D (Gi) day 9 vs day $10 \mathrm{p}=0.96$, one-way ANOVA.

(d) Stability of place cell representation calculated by population vector correlation between day 8 and day 9, day 9 and day 10 in D21 hM4D (Gi) or saline hM4D (Gi) animals. P = 0.023 D21 hM4D (Gi) day 8 and 9 vs Saline hM4D (Gi) day 8 and 9, p = 4.7 x10-6 D21 hM4D(Gi) day 8 and 9 vs Saline $\mathrm{hM} 4 \mathrm{D}(\mathrm{Gi})$ day 9 and 10, $\mathrm{p}=0.53$ Saline $\mathrm{hM} 4 \mathrm{D}(\mathrm{Gi})$ day 8 and 9 vs Saline hM4D(Gi) day 9 and 10, one-way ANOVA. $\mathrm{N}=10$ session pairs for D21 hM4D (Gi) day 8 and day9, 6 session pairs for saline hM4D (Gi) day 8 and day 9, 6 session pairs for saline hM4D (Gi) day 9 and day 10 .

\section{Discussion}

$\mathrm{mPFC}$, in particular ACC, has been implicated in the process of memory consolidation $[9,10]$.

During this process, coherent theta oscillations coupling between the hippocampus and mPFC facilitates the transfer of memory [25]. However, the exact content of memory carried by the neurons in ACC and whether it changes their properties during consolidation, have not been fully elucidated. In this study, we examined how representation of positional information is processed in the downstream of hippocampus. To this end, we recorded calcium activity over days from the ACC of freely moving mice. We found a population of neurons in ACC which shows location specific activity. It shares basic properties with hippocampal place cells such as location specific firing and directional selectivity [1-4]. However, unlike hippocampus place cells, the ACC place cells often have extra firing field. This may represent association of information from more than one location on the track. Indeed, mPFC including ACC is involved in the processing contextual information and such association of multiple locations is likely to play a role. 
In addition, we found that ACC place cells gradually increased over days in a manner requiring hippocampal activity for the formation. However, once the ACC place map is formed after 9 days, ACC no longer required hippocampal activity to fire and to exhibit a place cell map. This property is reminiscent of systems consolidation process of episodic memory, where hippocampus is required for initial formation of memory but not for recall of remote memory $[11,12,26]$. The memory consolidation theory predicts hippocampal activity after the memory events induces cortical

330 plasticity and consolidates memory in cortical circuit[11]. After memory consolidation, hippocampus 331 is no longer required for recall of remote memory[11]. The same analogy can be applied to the place 332 cells in both hippocampus and ACC. At this point, it is not clear from which brain regions the spatial 333 information is generated under hippocampal inactivation. ACC is bidirectionally connected to other 334 cortical regions such as retrosplenial and entorhinal cortexes, both of which are implicated in processing spatial information $[14,27,28]$. It is therefore possible that spatial information arriving from these cortexes as well other regions can bypass the requirement of hippocampus.

In conclusion, we found that ACC place cells have both shared and unique properties with 338 hippocampal counterparts. Further study is required to elucidate how their formation depends on the 339 hippocampus, but they are maintained without hippocampal activity once they are formed. It will be intriguing to test if these cells indeed overlap with engram cells as defined by $c$-fos expression [6].

\section{ACKNOWLEDGEMENTS}

343 We thank Dr. Adam Z. Weitemier for their comments on the manuscript. This work was supported

344 by RIKEN, Grant-in-Aid for Scientific Research A JP19H01010, Grant-in-Aid for Scientific

345 Research on Innovative Area "Foundation of Synapse and Neurocircuit Pathology" JP22110006,

346 "Principles of memory dynamism elucidated from a diversity of learning systems" JP90466037, and

347 "Constructive understanding of multi-scale dynamism of neuropsychiatric disorders" JP18H05434

348 from MEXT, and Human Frontier Science Program RGP0022/2013 to Y.H. and JP20K22686 from

349 MEXT and the Sasakawa Scientific Research Grant from The Japan Science Society to A.B. 


\section{Reference}

1. Eichenbaum, H., et al., The hippocampus, memory, and place cells: is it spatial memory or a memory space? Neuron, 1999. 23(2): p. 209-26.

2. O'Keefe, J.N., Lynn, The Hippocampus as a Cognitive Map. Oxford: Clarendon Press, 1978.

3. Moser, M.B., D.C. Rowland, and E.I. Moser, Place cells, grid cells, and memory. Cold Spring Harb Perspect Biol, 2015. 7(2): p. a021808.

4. Cobar, L.F., L. Yuan, and A. Tashiro, Place cells and long-term potentiation in the hippocampus. Neurobiol Learn Mem, 2017. 138: p. 206-214.

5. Ziv, Y., et al., Long-term dynamics of CA1 hippocampal place codes. Nat Neurosci, 2013. 16(3): p. 2646.

6. Ghandour, K., et al., Orchestrated ensemble activities constitute a hippocampal memory engram. Nat Commun, 2019. 10(1): p. 2637.

7. Gauthier, J.L. and D.W. Tank, A Dedicated Population for Reward Coding in the Hippocampus. Neuron, 2018. 99(1): p. 179-193 e7.

8. Sato, M., et al., Distinct Mechanisms of Over-Representation of Landmarks and Rewards in the Hippocampus. Cell Rep, 2020. 32(1): p. 107864.

9. Bontempi, B., et al., Time-dependent reorganization of brain circuitry underlying long-term memory storage. Nature, 1999. 400(6745): p. 671-5.

10. Teixeira, C.M., et al., Involvement of the anterior cingulate cortex in the expression of remote spatial memory. J Neurosci, 2006. 26(29): p. 7555-64.

11. Frankland, P.W., et al., The involvement of the anterior cingulate cortex in remote contextual fear memory. Science, 2004. 304(5672): p. 881-3.

12. Kitamura, T., et al., Engrams and circuits crucial for systems consolidation of a memory. Science, 2017. 356(6333): p. 73-78.

13. Vogt, B.A. and G. Paxinos, Cytoarchitecture of mouse and rat cingulate cortex with human homologies. Brain Struct Funct, 2014. 219(1): p. 185-92.

14. Hoover, W.B. and R.P. Vertes, Anatomical analysis of afferent projections to the medial prefrontal cortex in the rat. Brain Struct Funct, 2007. 212(2): p. 149-79.

15. Laubach, M., et al., What, If Anything, Is Rodent Prefrontal Cortex? eNeuro, 2018. 5(5).

16. Fujisawa, S., et al., Behavior-dependent short-term assembly dynamics in the medial prefrontal cortex. Nat Neurosci, 2008. 11(7): p. 823-33.

17. Hyman, J.M., et al., Contextual encoding by ensembles of medial prefrontal cortex neurons. Proc Natl Acad Sci U S A, 2012. 109(13): p. 5086-91.

18. Ma, L., et al., A Quantitative Analysis of Context-Dependent Remapping of Medial Frontal Cortex Neurons and Ensembles. J Neurosci, 2016. 36(31): p. 8258-72.

19. Mashhoori, A., et al., Rat anterior cingulate cortex recalls features of remote reward locations after disfavoured reinforcements. Elife, 2018. 7.

20. Burton, B.G., et al., Lesion of the ventral and intermediate hippocampus abolishes anticipatory activity in the medial prefrontal cortex of the rat. Behav Brain Res, 2009. 199(2): p. 222-34.

21. Low, R.J., Y. Gu, and D.W. Tank, Cellular resolution optical access to brain regions in fissures: imaging medial prefrontal cortex and grid cells in entorhinal cortex. Proc Natl Acad Sci U S A, 2014. 111(52): p. 18739-44.

22. Hafting, T., et al., Microstructure of a spatial map in the entorhinal cortex. Nature, 2005. 436(7052): p. 801-6.

23. Rubin, A., et al., Hippocampal ensemble dynamics timestamp events in long-term memory. Elife, 2015. 4.

24. Thompson, K.J., et al., DREADD Agonist 21 Is an Effective Agonist for Muscarinic-Based DREADDs in Vitro and in Vivo. ACS Pharmacol Transl Sci, 2018. 1(1): p. 61-72. 
25. Xing, B., M.D. Morrissey, and K. Takehara-Nishiuchi, Distributed representations of temporal stimulus

\section{Materials and methods}

\section{Subjects}

All experiments were conducted in accordance with institutional guidelines and protocols approved conducted on 10-24 weeks old male TRE-G-CaMP7 x CaMKII $\alpha$-tTA double transgenic mice [8].

431 To remove crosstalk from neighboring ROIs, clustering score of each ROI is calculated as follows.

432 After ROIs (red) were identified using a custom MATLAB routine (see method), 5-time enlarged 433 ROIs (by area, yellow) were made. For a given frame of $\mathrm{Ca} 2+$ images, the location of pixels with top $20 \%$ brightness were detected. The proportion of the pixels within the original ROI is defined as the clustering score for each frame. Low clustering score indicates high likeliness of crosstalk of neighboring ROI.

\section{Histology}

438 Mice were perfused transcardially with phosphate-buffered saline (PBS) followed by 4\%

439 paraformaldehyde in PBS. Brains were extracted and post-fixed in formalin for an additional day, and were transferred to a PBS for an additional day. Brains were then sliced in $50 \mu \mathrm{m}$ sections 
using a microslicer (Dosaka). For immunohistochemistory, sliced tissue sections were incubated in $0.1 \mathrm{M}$ Tris- $\mathrm{HCl}, 0.15 \mathrm{M} \mathrm{NaCl}, 0.5 \%$ Triton-X and $5 \%$ blocking reagent (Roche) and rabbit antiGFP antibody ( A11122, Thermo Fisher Scientific, 1:500) and incubated overnight at $4{ }^{\circ} \mathrm{C}$. After rinsing with PBS 3 times for 15 min each, sliced tissue sections were subsequently incubated with AlexaFluor 488 (A11008, Thermo Fisher Scientific, 1:500) conjugated secondary antibodies. Sliced tissue sections were then washed in PBS 3 times for 15 min, mounted, and coverslipped with mounting medium with Hoechst 33258 (\#382061, Calbiochem ). Fluorescence images were taken by confocal microscopy (Olympus FLUOVIEW FV1500) [29].

\section{Surgery for calcium imaging from Hippocampal CA1}

451 Mice were anesthetized with isoflurane in ambient air (3\% induction, $1.5 \%$ maintenance) and placed in a stereotaxic frame. A stainless-steel head plate $(25 \mathrm{~mm}$ length, $4 \mathrm{~mm}$ width, $1 \mathrm{~mm}$ thickness) with a circular opening ( $7 \mathrm{~mm}$ inner diameter and $10 \mathrm{~mm}$ outer diameter, the center is 2.5 $\mathrm{mm}$ off relative to the middle of the long side of the plate) was affixed to the skull using dental cement. The center of the opening was targeted at $2 \mathrm{~mm}$ posterior to the bregma and $2 \mathrm{~mm}$ lateral to the left from the midline. The cement was placed onto the skull such that it covered the entire skull, including the three anchor screws, except for the area of skull inside the opening of the head plate.

Several days later, we used a trephine drill to remove a circular part of the left skull centered 2.0 $\mathrm{mm}$ posterior, $2.0 \mathrm{~mm}$ lateral, and $2.0 \mathrm{~mm}$ deep from bregma. We removed the dura and cortex above the CA1 by suction with a 25 or 27 gauge blunt needle while constantly washing the exposed tissue with sterile cortex buffer $(123 \mathrm{mM} \mathrm{NaCl}, 5 \mathrm{mM} \mathrm{KCl}, 10 \mathrm{mM}$ glucose, $2 \mathrm{mM} \mathrm{CaCl} 2,2 \mathrm{mM}$ $\mathrm{MgCl}_{2}, 10 \mathrm{mM}$ HEPES, $\mathrm{pH}$ 7.4). We then implanted a metal guide tube with its window just dorsal to CA1 region and sealed the space between the skull and guide tube using dental acrylic [23].

\section{Surgery for calcium imaging from ACC}

466 A stainless-steel head plate was affixed to the skull as described above at $1 \mathrm{~mm}$ anterior to the bregma centered at midline. The dura was removed to provide access to the longitudinal fissure. Several days later, a microprism comprised of a right angle microprism (1 mm x $1 \mathrm{~mm} \times 1 \mathrm{~mm})$ bonded to GRIN lens (0.85-mm diameter, 3.3-mm length, Inscopix) was implanted. The face of the prism was aligned parallel to the fissure and positioned over the midline at the target $1.0-\mathrm{mm}$ anterior to bregma. After gently nudging the sinus laterally $(\sim 150 \mu \mathrm{m})$, the tip of the prism was positioned at the entrance to the fissure. Then the micromanipulator was used to vertically lower the prism into the subdural space in the longitudinal fissure so that its front faces against the medial surface of the left ACC. We then sealed the space between the skull and lens using dental cement and adhesive bond. The exposed lens was sealed with Silicone adhesive (Kwik-Sil, World Precision Instruments) and dental cement [21]. 
For hippocampal imaging, four weeks after the surgery described above, a gradient refractive index lens (GRINtech $\mathrm{GmbH}, 0.44$ pitch length, $0.47 \mathrm{NA}$ ) was fixed in the guide tube using ultravioletcuring adhesive (Norland, NOA 81). For ACC imaging, the silicone adhesive was removed to expose prism lens. We lowered the integrated microscope (nVistaHD, Inscopix) toward the GRIN lens until we saw G-CaMP7 fluorescence using the microscope's LED light source (0.12-0.24 $\mathrm{mW})$. After finding a suitable imaging site, we attached the microscope's base plate to the skull using dental acrylic darkened with black acrylic paint. This plate stayed with the mouse even after the microscope was detached to ensure reproducibility of imaging site. Before each behavior experiment, the microscope was attached to the base plate under light isoflurane anesthesia. The imaging session was started at least after 15 min recovery in the home cage.

In each session of the behavioral experiment, the animals were placed first in a square track (context S1), then an open arena (context $\mathrm{O}$ ) with different wall patterns, and then again in the square track (context S1') for 10 min each with 5 min interval. Context $\mathrm{S} 1$ consists of a square track of four divisions (each $50 \mathrm{~cm}$ long $\mathrm{x} 5 \mathrm{~cm}$ wide) and side walls $(8 \mathrm{~cm}$ tall). A wall was placed at one of the corners to separate the ends. The animals were trained to run back-and-forth between the two ends for a sucrose tablet (\#1811251 Sucrose Rewards Tablet, Test Diet), given when the animal reached one end. In the context $\mathrm{O}(50 \times 50 \mathrm{~cm}$ with wall of $25 \mathrm{~cm}$ tall), the rewards were randomly thrown into the open arena. Immediately before the beginning of each session, the equipment was wiped with differently scented paper towels ( $80 \%$ ethanol for context $\mathrm{S}$ and $0.5 \%$ acetic acid for context $\mathrm{O}$ ). Before beginning the imaging, mice were food restricted and they were exposed to context $\mathrm{O}$ and context $\mathrm{S}$ as pre-training for 3 days. After pre-training, one S1-O-S1' session per day was performed during calcium imaging and repeated for 9 days. On day 1 , mice were let freely move for $10 \mathrm{~min}$ in each context without any food reward. After day 1 pre-training, foods in their home cage are removed. In day 2 and 3, food reward was given as described above. After each day 2 day 3 of pre-training and each daily imaging, mice got 1-3 $\mathrm{g}$ food to keep their weight. We recorded a total 5 mice from hippocampus and a total 4 mice from ACC [5, 23].

\section{DREADD}

For DREADD experiments, DREADD agonist 21 (D21; Cayman Chemical Company, \#18907), an alternative to CNO, was made in a stock solution of $5 \mathrm{mg} / \mathrm{ml}$ in DMSO and then diluted in saline to desired concentration $(0.05 \mathrm{mg}$ or $0.1 \mathrm{mg} / \mathrm{ml})$. D 21 was injected intraperitoneally at $1 \mathrm{mg} / \mathrm{kg} 20$ min before the behavioral experiment.[30]

\section{Processing of Calcium imaging data}

We processed imaging data using Mosaic (Inscopix). To increase computation speed, we spatially down-sampled the data by a factor of four in each dimension. The down-sampled imaging data was motion corrected and transformed to relative changes in fluorescence, $\Delta \mathrm{F}(\mathrm{t}) / \mathrm{F} 0=(\mathrm{F}(\mathrm{t})-\mathrm{F} 0) / \mathrm{F} 0$, where $\mathrm{F} 0$ is the value for each pixel averaged over the entire time span of the calcium imaging movie. Finally, the movie was smoothed by applying disk average filter (disk radius: $3 \times 3$ pixels). 
518 We separately used un-filtered movie for calculation of each neuron's fluorescence intensity, and

519 filtered movie for neuron identification and calculating clustering score, respectively. (see 'Neuron

520 Identification' and 'Calcium Event Identification').

521 (Users Manual of Mosaic, Inscopix, 2014)

\section{Neuron Identification}

Regions-of-interest (ROIs) was identified using a custom MATLAB routine. First, intensity of each pixel is normalized by the mean value of all pixel in that frame of the filtered calcium imaging movie. Then, it adaptively thresholds each imaging frame to identify separable pockets of calcium activity, designated as blobs, on each frame. Blobs of activity were accepted at this stage of processing only if they approximate the size and shape of a neuron, as measured by their size (min $=250 \mu \mathrm{m}^{2}$ (30 pixels), $\max =1500 \mu \mathrm{m}^{2}(180$ pixels $)$ ), and the ratio of long to short axes $(\max =2)$, a metric used by the regionprops function of MATLAB we employ to reject jagged/strange shaped blobs. Signal traces of each listed blob are calculated over all frames of the movie. Finally, overlap of region among all blobs and correlation of activity (mean gray value of pixels in the blob) among all blobs were calculated respectively. Blob pairs with high spatial correlation $(r>0.4)$ and high signal correlation $(r>0.9)$ are considered as the same neuron. Smaller one of the two was used as ROI for further analysis [29].

\section{Calcium Event Identification}

537 We extracted $\Delta \mathrm{F} / \mathrm{F}$ traces of each ROIs from un-filtered calcium imaging movies. To remove crosstalk from neighboring ROIs, clustering score of each ROI is calculated as follows. After ROIs (red) were identified using a custom MATLAB routine, 5-time enlarged ROIs (by area, yellow) were made (Fig. S5). For a given frame of $\mathrm{Ca}^{2+}$ images, the location of pixels with top $20 \%$ brightness were detected. The proportion of the pixels within the original ROI is defined as the clustering score for each frame. Low clustering score indicates high likeliness of crosstalk of neighboring ROI. By excluding value with low clustering score from each ROI, crosstalk was removed. Second and third steps are performed in each frame of movie. Time points with $\Delta \mathrm{F} / \mathrm{F}$ signal $>1.5$ and clustering score $>0.4$ are detected as calcium event for each ROI.

\section{Neuron Registration}

548 Neuron registration occurred in two steps: session registration and neuron registration.

\section{Session registration}

550 Prior to mapping neurons between sessions, we determined how much the imaging window shifted 551 between sessions. In order to isolate consistent features of the imaging plane for each mouse (such 552 as vasculature or coagulated blood), we created a median projection of all of the frames of the 553 imaging movie for each recording session. One session ("registered session") was then registered 554 to a base session (first session) using the 'motion correction' function from the Mosaic and 
calculated transformation object was saved. This process of transformation was applied to each calcium imaging movie of all sessions, and saved for further analysis. [29]

\section{Neuron Registration}

Next, each ROI in the registered session was transformed to its corresponding location in the base session. Each neuron in the base session was then mapped to the neuron with the closest center-ofmass in the registered session, unless the closest neuron exceeded our maximum distance threshold of 4 pixels $(10 \mu \mathrm{m})$. The spatial correlation between each pair of ROIsd are also calculated. We designated the base session ROI with the high spatial correlation $(r>0.6)$ as mapping to the registered session ROI. [29]

\section{Place cells and place field}

We used the following criteria to identify place cells and place fields in the open field and the square track, respectively.

\section{Place cells in open field}

To identify place cells in the open field, firing rate maps were obtained as follows. First, calcium events from each ROI were assigned to $16 \mathrm{~cm}$ x $16 \mathrm{~cm}$ spatial bins. Second, the number of calcium events in each bin was divided by the occupancy time of that bin.

Then we computed the spatial information using the firing rate maps of each cell, as previously described [31]

Where $r_{i}$ is the calcium event rate of the neuron in the $i^{\text {th }}$ bin; $P_{i}$ is the probability of the mouse being in the $i^{\text {th }}$ bin (time spent in $i^{\text {th }}$ bin/total session time); $\bar{r}$ is the overall mean calcium event rate; and $i$ running over all the bins. We then performed 1000 permutation shuffles, and calculated the spatial information for each shuffle. This yielded the $\mathrm{p}$ value of the measured information relative to the shuffles. Cells with $\mathrm{p}<0.05$ and mean firing rates higher than $0.1 \mathrm{~Hz}$ were classified as place cell.

To detect place fields in the open field, we make firing rate maps with $2.5 \mathrm{~cm} \times 2.5 \mathrm{~cm}$ spatial bins, normalized firing rate of each bin by maximum value in the map, and smoothed the normalized map with 1 SD Gaussian kernel. Next, we made binary map based on the firing rate of each bin (spatial bin with firing rate>0.2 is 1 , other spatial bins are 0). Connected bins in this binary map are detected by using MATLAB bwlabel function (Pixel connectivity $=8$-connected). Each connected bin is considered as individual place field. Place field which contains bin with maximum firing rate is designated as main place field, and other place fields as extra place field. [23] [32] 
To identify place cells in the square track, we employed reliability, which measured the consistency of a cell to fire on its preferred location along the track (Supplementary Fig. 2) [33]. We calculated reliability in the following manner. First, we divided track into 72 spatial bins $(2.8 \mathrm{~cm}$ each), and exclude the first and the last 5 bins where food rewards were given. Spatial activity in each lap was transformed into binarized vectors, in which 1 and 0 represent the presence and absence of a calcium events, respectively. Third, we calculated the correlation value between each lap and the mean of all correlation values. Finally, the locations of calcium events in the binarized vector are shuffled randomly 1000 times, then reliability of each shuffled data was calculated. Cells with significant reliability $(\mathrm{p}<0.05)$ are considered as place cell in the square track (Supplementary Fig. 2). Number of running lap with calcium event of all cells and population vector correlations between a session and all other sessions are calculated to exclude session without spatial activity. The sessions with mean number of running with calcium event lap $<1.5$ or mean population vector correlation $<0.005$ are excluded from analysis. We separately considered place cells for forward and backward running directions.

To detect place fields in the square track, we normalized the firing rate map by maximum value, smoothed the normalized map with 1 SD Gaussian kernel, and converted the smoothed map to binary map (spatial bin with firing rate $>0.2$ is 1 , other spatial bins are 0 ). Connected bins in this binary map are detected, and each connected bin is considered as individual place field. Place field which contains bin with maximum firing rate is designated as main place field, and other place fields as extra place field[23].

\section{Selectivity index}

We used the following calculation to determine the selectivity index of each cell: |(activity in context $\mathrm{S} 1$ - activity in context $\mathrm{O}$ ) / (activity in context $\mathrm{S} 1$ + activity in context $\mathrm{O}) \mid$.

\section{Population vector correlation}

To determine the level of similarity between representations of the different sessions, we calculated the mean population vector correlation between them [34]. For each spatial bin (excluding the last 5 bins at both ends of the tracks) we defined the population vector as the mean event rate for each neuron given that bin's firing rate. We computed the correlation between the population vector in one session with that of the matching location in the other session, and averaged the scores over all positions. [23]

\section{Statistics}

Data are expressed as means \pm SEM unless stated otherwise. All statistical tests were performed using MATLAB. One-way ANOVA was used for group/pair-wise comparisons. Where appropriate, Student's paired t-tests or Wilcoxon rank sum tests was conducted. The null hypothesis was rejected at the $\mathrm{P}<0.05$ level. 


\section{Bayesian decoder}

629 We used a Naive Bayes Classifier [5, 23, 35] to estimate the mouse location based on neuron calcium activity. The computation of the conditional probability for the subject to be at location $x$ is based on Bayes formula:

$$
\mathrm{P}(\mathrm{x} \mid \vec{n})=\mathrm{P}(\vec{n} \mid x) \mathrm{P}(x) / \mathrm{P}(\vec{n})
$$

$n$ is the population activity vector of length $N$ containing 1 at index $i$, when neuron $i$ is considered to be active and 0 when it is not. $P(x)$, the probability for the subject to be at position $x$ was obtained from the dwell time distribution at each spatial bin (bin size $=2.8 \mathrm{~cm}$ ). $P(\vec{n} \mid x)$, the conditional probability to observe $\vec{n}$ given the subject is at position $x$ was computed from the spatial map $P\left(n_{i} \mid x\right)$ of individual neurons $i$ assuming statistical independence between their activity [5].

$$
\mathrm{P}(\vec{n} \mid x)=\prod_{i=1}^{N} p\left(n_{i} \mid x\right)
$$

The overall probability to observe $\mathrm{n}$ is obtained from $P(\vec{n})=\sum_{j \in X} P\left(\vec{n} \mid x_{j}\right) \mathrm{P}\left(\vec{n} \mid x_{j}\right) \mathrm{P}\left(x_{j}\right)$, with $\mathrm{P}\left(x_{j}\right)$ normalized along the spatial dimension $X$. A reconstructed position of the subject is obtained from the peak position of the evaluated $P(x \mid \vec{n})$, Eq.1 with $\vec{n}=\vec{n}(\mathrm{t})$. This estimate is further refined taking into account the data from $d T=8$ frames before the current frame, assuming a negligible change in position of the animal within $d T$ :

$$
x_{r e c}=\operatorname{argmax} \prod_{j} \prod_{\delta t=0}^{d T} P\left(\vec{n}(t-\delta t) \mid x_{j}\right) P\left(x_{j}\right) / \mathrm{P}(\vec{n}(t-\delta t))
$$

The estimation error was calculated as the absolute difference between the real and the reconstructed positions. We trained the decoder with the subjects observed positions and activities of all place cells (place cells with top 6-15\% higher firing rate were used) during the first half time of the running period and estimated the trajectory for the following half time. The running time of 
a
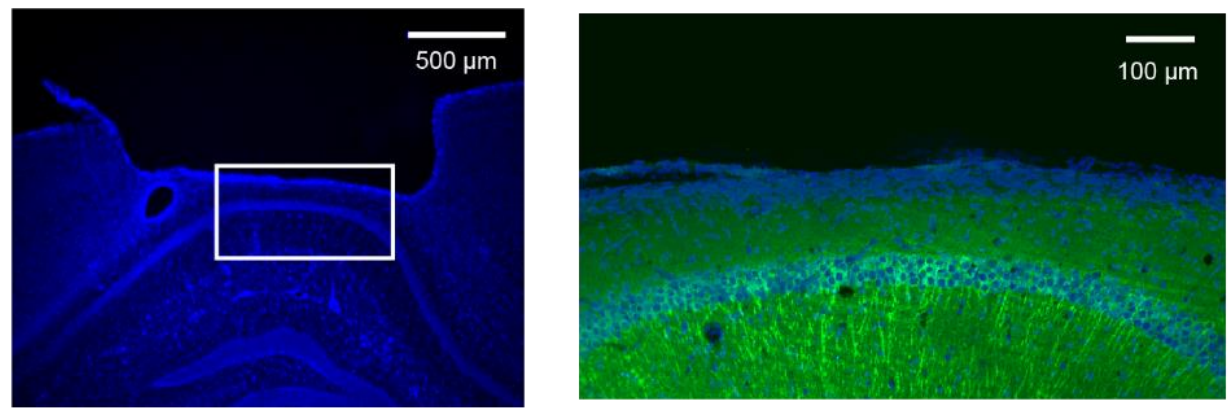

b
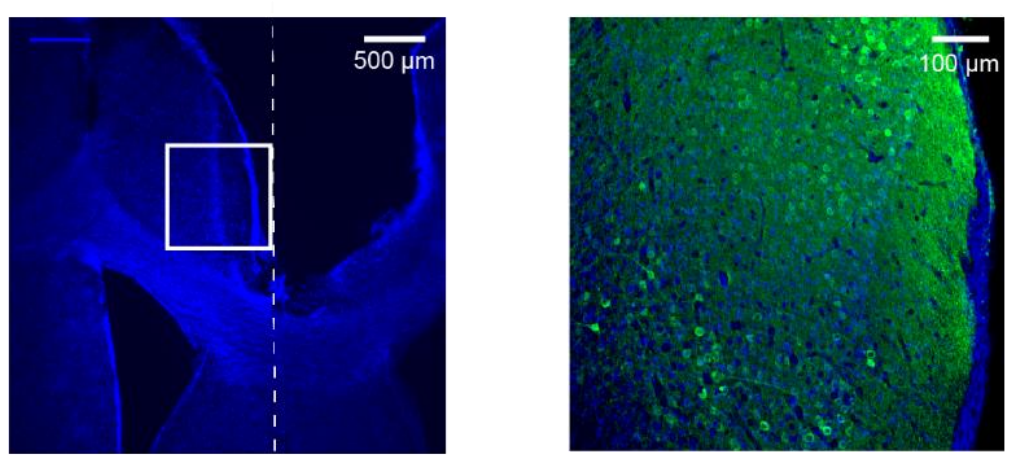

Bota et al. supple. 1

\section{Figure S1 Histological confirmation of imaging location.}

655 (a) Left, a coronal section stained with Hoechst 33258 (blue) and anti-GFP antibody (green) of 656 TRE-G-CaMP7 x CaMKII $\alpha$-tTA mouse after imaging from ACC. Right, A zoomed image from 657 rectangle area. Hoechst image was overlaid with G-CaMP7 immunofluorescence.

658 (b) A coronal section stained with Hoechst 33258 and anti-GFP antibody of TRE-G-CaMP7 x 659 CaMKII $\alpha$-tTA mouse after imaging from hippocampus. 


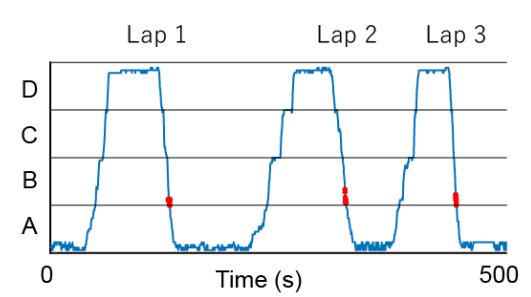

Cell \#23 Reliability $=0.92$

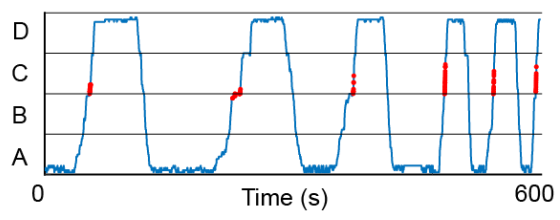

Lap 1

Lap 2
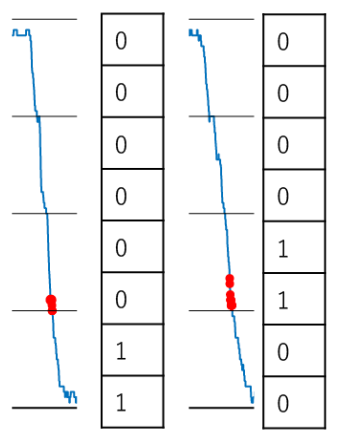

Correlation between each lap $\Rightarrow$ average (Reliability)

Bota et al. supple. 2

Cell \#78 Reliability $=0.51$

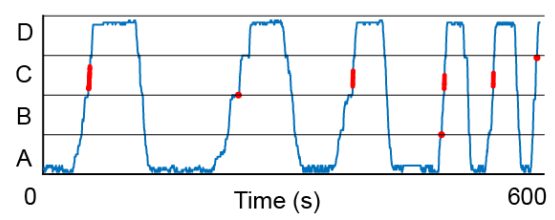

\section{Figure S2. Calculation of reliability of place cell.}

664 Examples of trajectory of mouse and calcium events of ACC place cells. Spatial activity in each lap was transformed into binarized vectors, in which 1 and 0 represent the presence and absence of a calcium events, respectively. We calculated the correlation value between each lap and the mean of
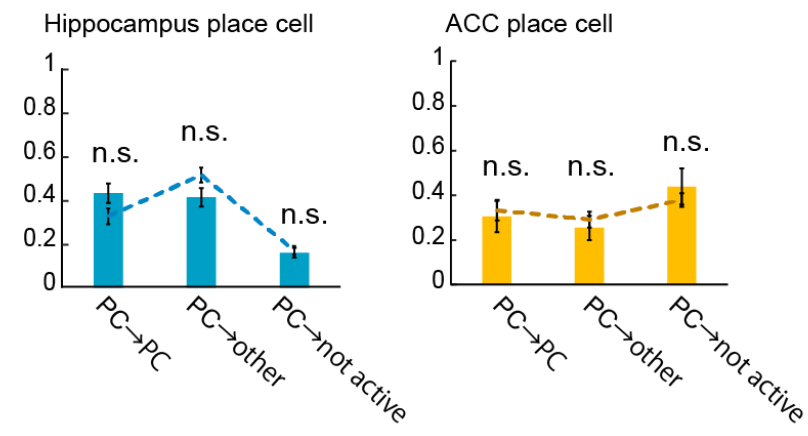

Bota et al. supple. 3 
Figure S3. Conversion of encoding mode of hippocampal and ACC place cells in context $\mathrm{O}$ and in context $S 1$.

Encoding mode of hippocampal or ACC place cells in context $\mathrm{O}$ was examined in context S1'.
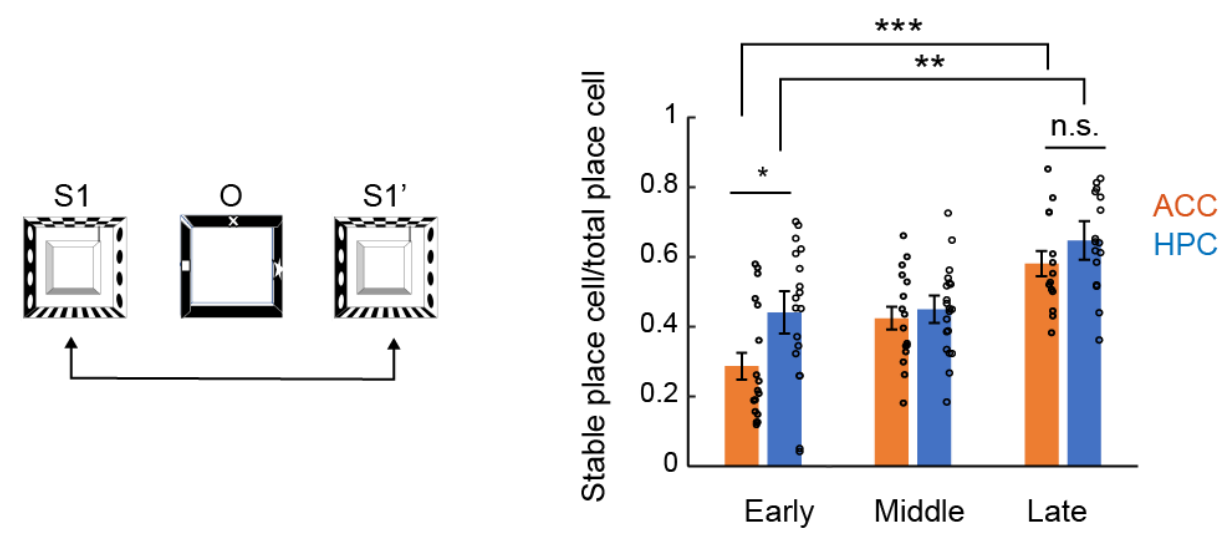

\section{Bota et al. supple. 4}

Figure S4. The fraction of stable place cells on the same day

680 Place cell stability calculated as the fraction of stable place cells relative to the number of total 681 place cells identified in each session that were compared. Cells in early phase (day 1-3), middle 682 phase (day 4-6) and late phase (day 7-9) were pooled respectively. $p=9.6 \times 10^{-6}$ (ACC early vs 683 ACC late), $\mathrm{p}=0.0015$ (hippocampus early vs hippocampus late), $\mathrm{p}=0.017$ (hippocampus early vs 684 ACC early), $\mathrm{p}=0.203$ one-way ANOVA. $\mathrm{N}=18$ session pairs in early, 20 session pairs in middle, 68516 session pairs in late for hippocampus. $\mathrm{N}=18$ session pairs in early, 16 session pairs in middle, 686 14session pairs in late for ACC. 

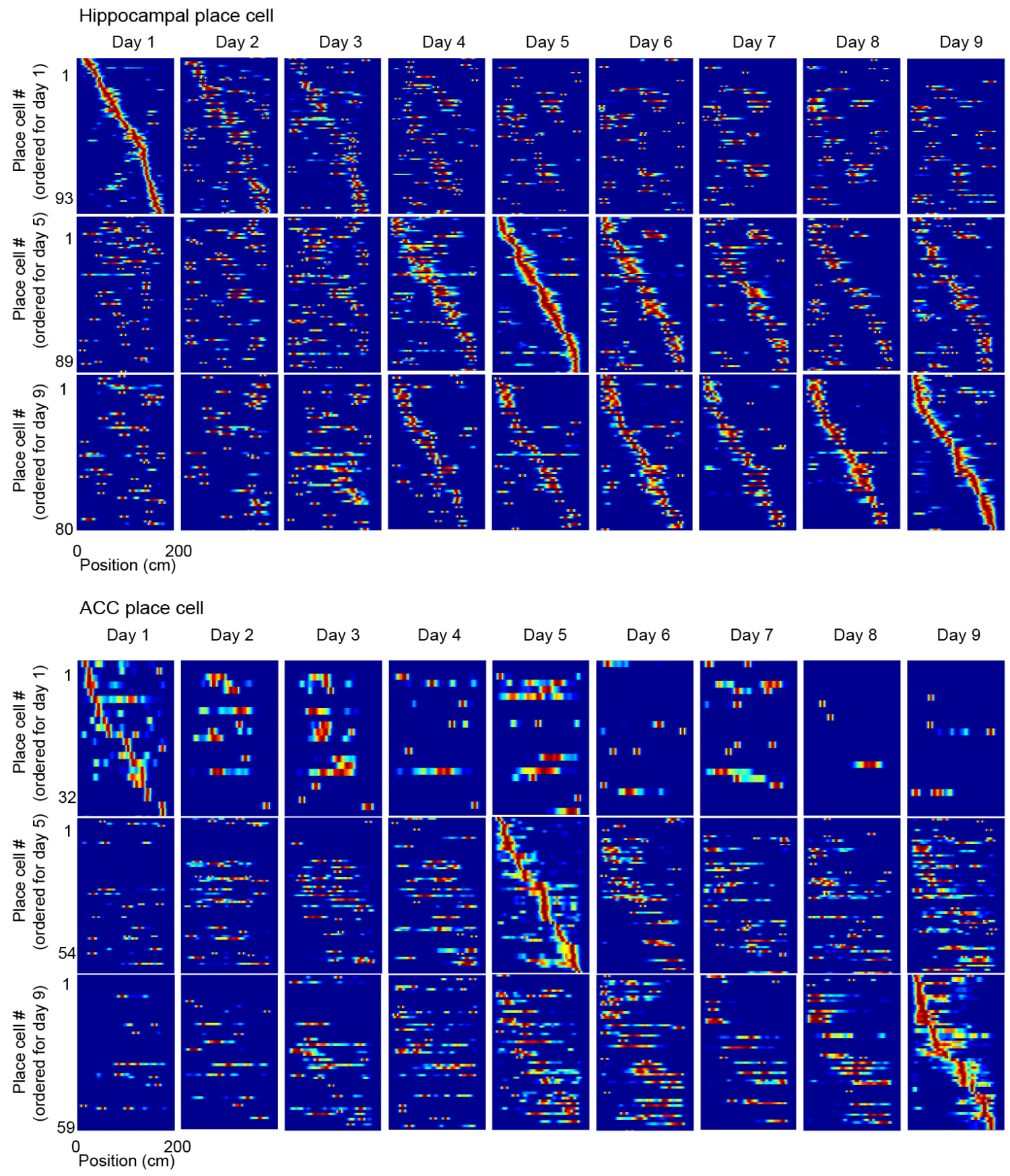

Day 3

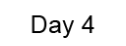

Day 5

Day 6

Day 7

Day 8

Day 9
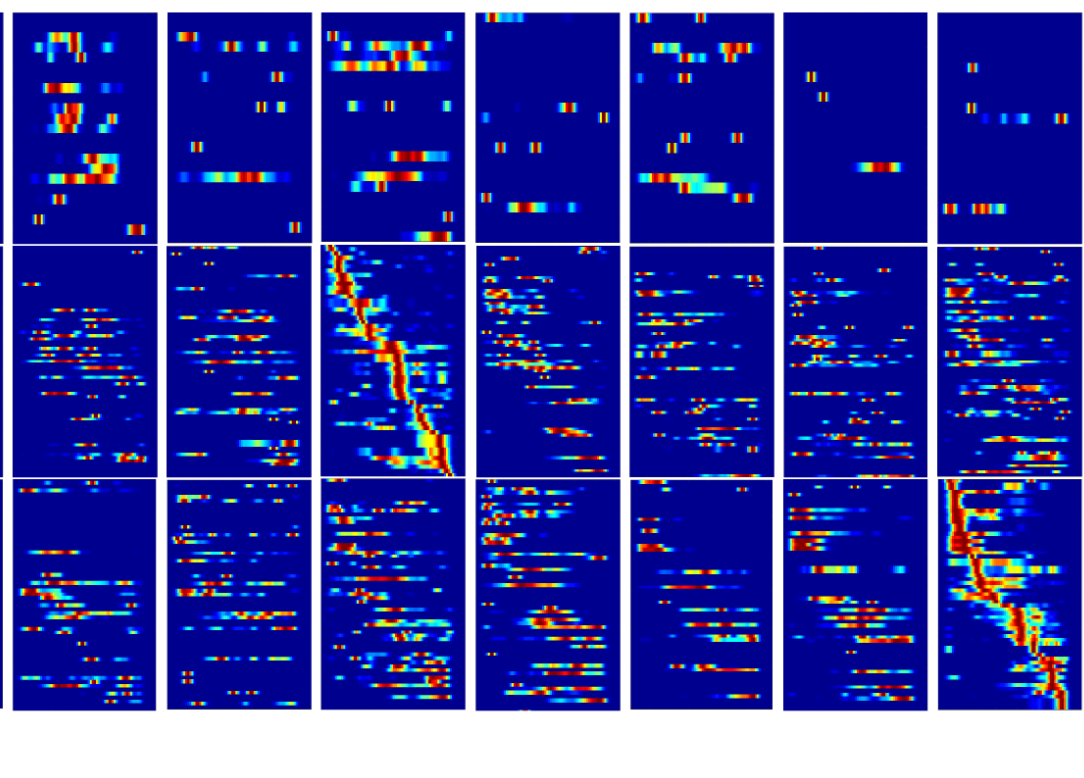

Bota et al. supple. 5

Figure S5. Remapping of place cells across days.

690 Place-field maps of hippocampal and ACC place cells ordered by their centroid positions on day 1

691 (top), day 5 (middle) or day 9 (bottom). 


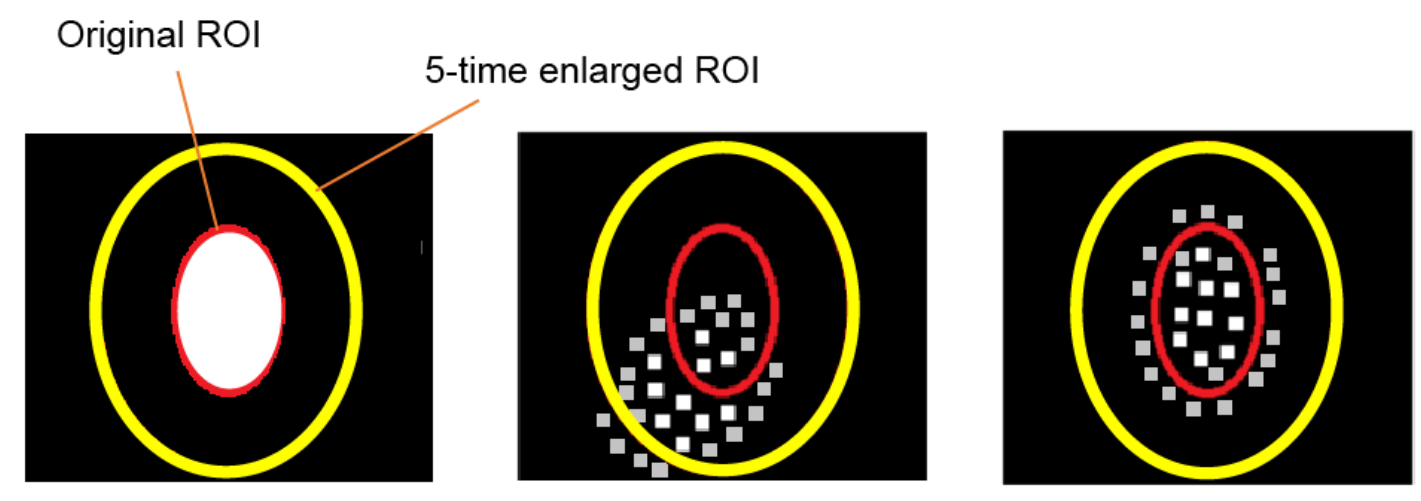

Bota et al. supple. 6

Figure S6. Clustering score.

696 To remove crosstalk from neighboring ROIs, clustering score of each ROI is calculated as follows. 697 After ROIs (red) were identified using a custom MATLAB routine (see method), 5-time enlarged 698 ROIs (by area, yellow) were made. For a given frame of $\mathrm{Ca}^{2+}$ images, the location of pixels with top $69920 \%$ brightness were detected. The proportion of the pixels within the original ROI is defined as the 700 clustering score for each frame. Low clustering score indicates high likeliness of crosstalk of 701 neighboring ROI. 